\title{
BRIEF SUMMARY OF PATHOGENESIS, DIAGNOSIS AND MANAGEMENT OF SPONTANEOUS CORONARY ARTERY DISSECTION
}

\author{
Algirdas Rẻkus ${ }^{1}$, Gediminas Jaruševičius ${ }^{2}$ \\ ${ }^{1}$ Faculty of Medicine, Lithuanian University of Health Sciences, Kaunas, Lithuania, \\ ${ }^{2}$ Department of Cardiology, Lithuanian University of Health Sciences, Kaunas, Lithuania
}

Keywords: coronary artery dissection, spontaneous, acute coronary syndrome, intramural hematoma.

\begin{abstract}
Summary
Spontaneous coronary artery dissection (SCAD) is a rare cause of acute coronary syndrome (ACS). It was first described 80 years ago. Pathogenetic mechanisms are most likely to be associated with intimas tear or bleeding vasa-vasorum, which resulting in intramural haemorrhage. SCAD typically occurs in young women who do not have coronary heart disease risk factors and who have acute coronary syndrome. Half of all SCAD presents with ST - elevation myocardial infarction (STEMI), while the rest with non - ST - elevation myocardial infarction (NSTEMI). The gold standard method for diagnosis is interventional coronary artery angiography. After the acute ischemic onset syndrome, most patients have a stable, benign clinical course, and eventually experience spontaneous vessel wall healing. Therefore, conservative treatment (a watchful strategy) is recommended as the initial treatment. For the majority of cases as interventional and surgical treatment in most cases seems to be suboptimal. In this extremely complex situation, several novel and attractive coronary interventions have been proposed. The risk factors, pathogenesis theories, diagnosis, management, prognosis of SCAD will be summarized in this review.
\end{abstract}

\section{Introduction}

As first described in 1931, spontaneous coronary artery dissection (SCAD) is a non-atherosclerotic coronary artery disease that manifests as acute coronary syndrome (ACS) or death (1). Primary or isolated SCAD is a disorder due to intramural hematoma (IMH) and / or dissection that obstructs coronary flow, which is not related to trauma nor is iatro- genic. During pregnancy and soon after childbirth, women are more susceptible to developing SCAD. According to Mayo clinic Registry data, about $17 \%$ of SCAD is associated with pregnancy (2). SCAD, previously considered rare, is responsible for $24-36 \%$ of myocardial infarction (MI) in women under the age of 50 and is now recognized as the most common cause of pregnancy-related MI (3). SCAD mainly affects women (92-98\% of cases), who are relatively young, 42-52 years old, and do not have traditional cardiovascular risk factors $(1,2)$. The prevalence of SCAD was $0,49 \%$ according to National Impatient Sample database, presenting more than 13 million patients admitted due to ACS between 2005 and $2015(4,5)$. However, these estimates probably not represent the real incidence of SCAD, as the disease could be often misdiagnosed, especially in the early era.

The aim of this review was to evaluate the risk factors, pathogenesis, diagnosis, management and prognosis of spontaneous coronary artery dissection.

\section{Methodology}

Data search in electronic scientific databases PubMed, Embase in the period 2016-2019 was performed. Search words: coronary artery dissection, spontaneous, acute coronary syndrome, intramural hematoma. A review of twentynine literature sources was conducted.

Risk factors and pathogenesis. SCAD has no pathognomic risk factors. It was noticed that, SCAD more often affects young women who do not have cardiovascular factors for cardiovascular disease (6-8). It is currently thought that risk factors for SCAD are multiple pregnancies (more than 4 times), administration of hormone replacement therapy, infertility therapy, connective tissue diseases, in particular, fibromuscular dysplasia, vascular Ehlers-Danlos syndrome, Marfan syndrome, Loeys-Dietz syndrome, emotional and physical stress $(2,9-12)$. Two major pathogenesis mecha- 
nisms of SCAD are currently described: (a) hypothesis of medial haemorrhage which indicates that haemorrhage in the artery wall is due to spontaneous rupture and intramural bleeding from vaso-vasorum is a major pathogenic mechanism; and (b) an intimate tearing hypothesis that disruption of the intimal interface creates an entry point for intramural hematoma accumulation in the false lumen and leads to arterial wall separation $(1,13-15)$.

Clinical presentation. Have been admitted SCAD patients complaints characteristic to ACS. Like cases of ACS, SCAD is characterized by chest, shoulder or upper abdominal pain, with or without radiation to the arm, nausea or vomiting, neck irradiation, sweating, dyspnoea, and rarely back pain, dizziness, fatigue, headache, fainting (1). Symptoms vary in severity from mild to severe, and cardiac dysfunction can cause arrhythmias, cardiogenic shock, heart failure $(16,17)$.

Diagnosis. SCAD manifest as ACS and thus usually is preliminary diagnosed by ECG and laboratory tests (series of troponin tests). Half of all patients have been diagnosed with ST - elevation myocardial infarction (STEMI), the rest - with non - ST - elevation myocardial infarction (NSTEMI) $(1,2,18)$. SCAD is diagnosed in most patients during coronary angiography (19). Indications for coronary artery angiography are ordinary. In 2014, Saw et al. (20) have developed 3 types of SCAD classification based on angiography (table 1). Non-invasive procedures, such as computed tomography and magnetic resonance coronary angiography, have limitations and are not used for SCAD diagnosis.

Management. Generally, the main treatment strategies for SCAD are coronary artery bypass surgery, percutaneous coronary intervention (PCI), medical therapy, and active surveillance. The choice of these treatment strategies still represents a debatable issue and may depend on individual clinical symptoms, the localization and spread of the lesion, the experience of the treating institution, and previous relapses.

Conservative medical therapy alone is preferred in stable patients. Medical therapy consisted of aspirin, P2Y12 inhibitors (clopidogrel) and beta blockers, followed by angiotensin converting enzyme inhibitors, calcium channel blockers, nitrovasodilators, and angiotensin II receptor blockers, for stent or bioresorbable vascular scaffold strategies, dual antiplatelet therapy is generally prescribed, usually for 12 months (21). In general, long-term results for SCAD patients are excellent, and most patients can safely receive medical therapy. Several retrospective case series have reported that spontaneous angiographic healing occurred in $73-97 \%$ of cases when repeated angiography was performed (1). However, SCAD can be life-threatening because some patients experience early or late complications, including SCAD of other coronary vessels. Therefore, further testing and optimal monitoring strategies have to be defined $(22,23)$. Currently, SCAD PCI and surgical treatment algorithm have been proposed to simplify the course of intervention (figure 1). Revascularization in SCAD patients is very difficult due to subcutaneous damage to coronary vessels (24). Although stenting is usually delayed, the urgency of revascularisation depends on clinical status and hemodynamic stability and urgent PCI is usually successful in life-threatening situations (25). Indications for revascularisation include complete vessel occlusion, left main involvement, ongoing ischemia, recurrent chest pain and haemodynamic instability, as well as sustained ventricular arrhythmias.

Coronary artery bypass grafting (CABG) is commonly used as a rescue strategy in failure of PCI for SCAD patients $(24,26)$. In case of diffuse left main dissection the literature on the CABG approach is limited by clinical cases. The short-term outcomes of CABG treatment appear to be good, but the long-term coronary artery graft failure has been observed. Most likely due to spontaneous coronary artery wall healing. It has been suggested that both venous and arterial conduits may fail when the dissected artery has healed (2). Therefore, $\mathrm{CABG}$ is reserved for multivascular dissection and extreme cases of hemodynamic instability.

Alternative treatment for SCAD has been proposed performing fenestrations. Motreff et al. (27) conducted a study evaluating the efficacy of fenestrations. The intima was cut with a scoring or a cutting balloon inducing intramural hematoma decompression, excellent long-term results have been reported (27). It seems like cutting balloon angioplasty before stent placement prevents longitudinal extension of intramural hematoma and helps to avoid unnecessary stent implantation.

Prognosis. According to the largest contemporary cohort study, hospital mortality from SCAD was 4.2 percent (4). In a retrospective study including 87 patients, a SCAD relapse rate of 17 percent was observed (28). Hospital prognosis

Table 1. SCAD angiographic classification

\begin{tabular}{|c|l|}
\hline Type 1 & $\begin{array}{l}\text { Type 1 shows a classic appearance with contrast staining of the } \\
\text { arterial wall and multiple lumens }\end{array}$ \\
\hline Type 2 & $\begin{array}{l}\text { Type 2 represents diffuse stenosis of different weights and } \\
\text { lengths }\end{array}$ \\
\hline Type 2A & $\begin{array}{l}\text { Type 2A stenosis is restricted by the normal proximal and distal } \\
\text { lumen }\end{array}$ \\
\hline Type 2B & $\begin{array}{l}\text { Type 2B stenosis extends to the distal tip of the artery } \\
\text { Type 3 }\end{array}$ \\
$\begin{array}{l}\text { Type 3 represents localized or tubular stenosis that is difficult to } \\
\text { distinguish from atherosclerosis }\end{array}$ \\
\hline
\end{tabular}


is generally great for people undergoing conservative treatment, CABG or percutaneous coronary intervention $(28,29)$.

\section{Conclusion}

Spontaneous coronary artery dissection defined as the nontraumatic and non-aetrogenic separation of the coronary artery wall. It is a rare cause of acute coronary syndrome. Although rare, SCAD could be considered in young women presenting with ACS, even if there is no cardiovascular risk factors. The gold standard for diagnosing SCAD remains percutaneous coronary artery angiography. Despite improved evidence, the management of SCAD remains based on individual experience considering patients status and haemodynamic stability.

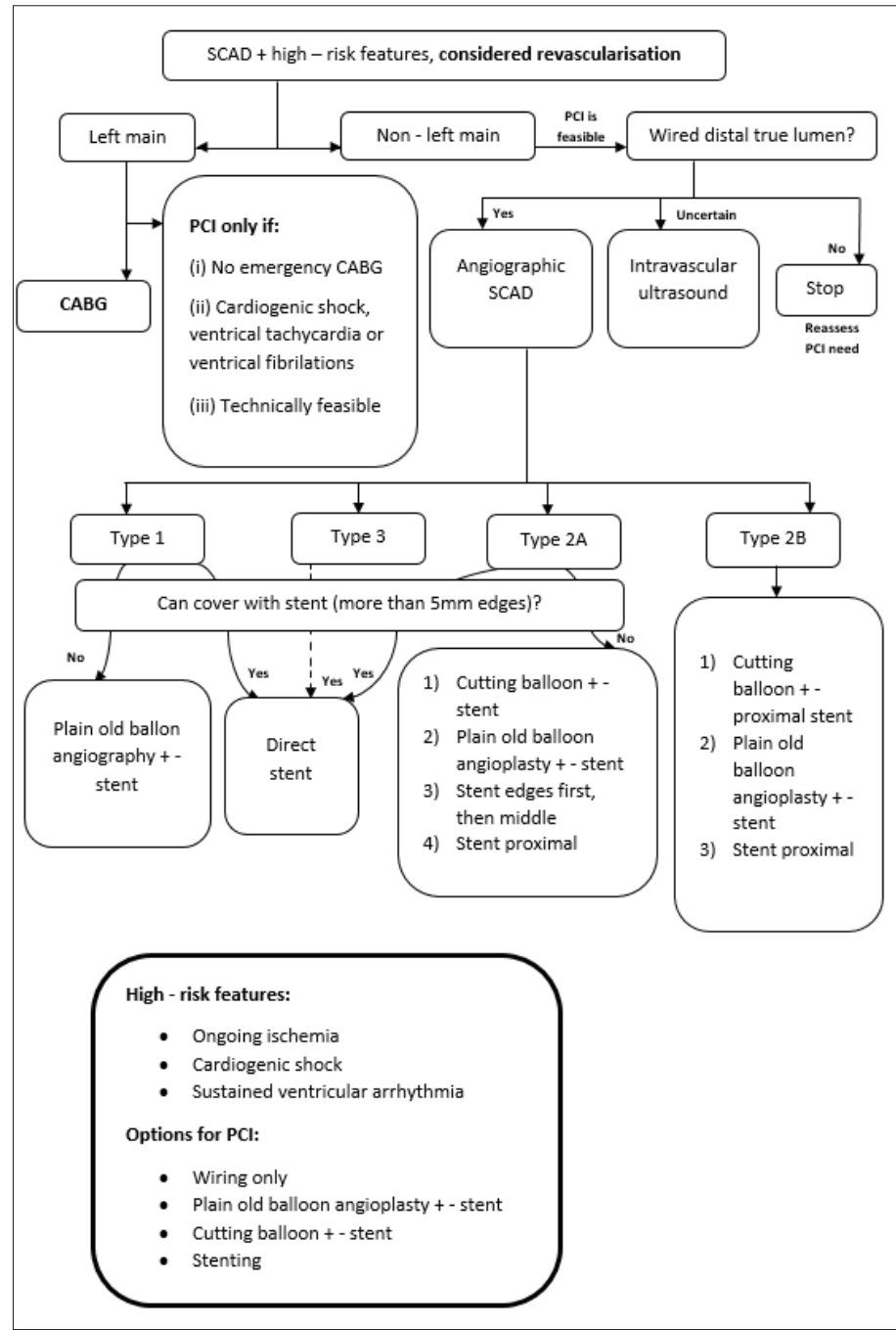

Figure 1. Suggested algorithm for SCAD PCI and CABG treatment. Adapted from: Jacqueline Saw. Natural history of spontaneous coronary artery dissection: to stent or not to stent? EuroIntervention. 2019 Jan 18;14(13):1353-6.2019;14:1353-6.

\section{References}

1. Saw J, Mancini GBJ, Humphries KH. Contemporary Review on Spontaneous Coronary Artery Dissection. J Am Coll Cardiol 2016 Jul 19;68(3):297-312. https://doi.org/10.1016/j.jacc.2016.05.034

2. Tweet MS, Hayes SN, Codsi E, Gulati R, Rose CH, Best PJM. Spontaneous Coronary Artery Dissection Associated With Pregnancy. J Am Coll Cardiol 2017 Jul 25;70(4):426-35.

https://doi.org/10.1016/j.jacc.2017.05.055

3. Rashid HNZ, Wong DTL, Wijesekera H, Gutman SJ, Shanmugam VB, Gulati R, et al. Incidence and characterisation of spontaneous coronary artery dissection as a cause of acute coronary syndrome - A single-centre Australian experience. Int J Cardiol 2016 Jan 1;202:336-8. https://doi.org/10.1016/j.ijcard.2015.09.072

4. Krittanawong C, Kumar A, Virk HUH, Yue B, Wang Z, Bhatt DL. Trends in Incidence, Characteristics, and InHospital Outcomes of Patients Presenting With Spontaneous Coronary Artery Dissection (From a National Population-Based Cohort Study Between 2004 and 2015). Am J Cardiol 2018 Nov 15;122(10):1617-23. https://doi.org/10.1016/j.amjcard.2018.07.038

5. Quadri G, Tomassini F, Cerrato E, Varbella F. First reported case of magnesium-made bioresorbable scaffold to treat spontaneous left anterior descending coronary artery dissection. Catheter Cardiovasc Interv [Internet]. 2017 [cited 2019 Dec 22];90(5):768-72. Available from: http://onlinelibrary.wiley.com/doi/abs/10.1002/ ccd. 27214

https://doi.org/10.1002/ccd.27214

6. Mkoko P, Pandie S, Ntsekhe M. Recurrent idiopathic spontaneous coronary artery dissection. S Afr Med J 2019 Jun 28;109(7):477-479-479. https://doi.org/10.7196/SAMJ.2019.v109i7.13997

7. Lindor RA, Tweet MS, Goyal KA, Lohse CM, Gulati R, Hayes SN, et al. Emergency Department Presentation of Patients with Spontaneous Coronary Artery Dissection. J Emerg Med 2017 Mar 1;52(3):286-91. https://doi.org/10.1016/j.jemermed.2016.09.005

8. Ghaffarian K, Furin M, Bassett R. STEMI in a young female? Consider spontaneous peripartum coronary artery dissection. Am J Emerg Med 2017 Feb 1;35(2):381. e1-381.e4.

https://doi.org/10.1016/j.ajem.2016.08.064

9. Vijayaraghavan Ram, Verma Subodh, Gupta Nandini, Saw Jacqueline. Pregnancy-Related Spontaneous Coronary Artery Dissection. Circulation 2014 Nov 18;130(21):1915-20.

https://doi.org/10.1161/CIRCULATIONAHA.114.011422

10. Sharma S, Kaadan MI, Duran JM, Ponzini F, Mishra S, Tsiaras SV, et al. Risk Factors, Imaging Findings, and 
Sex Differences in Spontaneous Coronary Artery Dissection. Am J Cardiol 2019 Jun 1;123(11):1783-7.

https://doi.org/10.1016/j.amjcard.2019.02.040

11. Henkin S, Negrotto SM, Tweet MS, Kirmani S, Deyle DR, Gulati R, et al. Spontaneous coronary artery dissection and its association with heritable connective tissue disorders. Heart 2016 Jun 1;102(11):876.

https://doi.org/10.1136/heartjnl-2015-308645

12. Adams H, Paratz E, Somaratne J, Layland J, Burns A, Palmer S, et al. Different patients, different outcomes: A case-control study of spontaneous coronary artery dissection versus acute coronary syndrome. J Intervent Cardiol 2018;31(1):41-7. https://doi.org/10.1111/joic.12447

13. Al-Hussaini A, Adlam D. Spontaneous coronary artery dissection. Heart. 2017 Jul 1;103(13):1043-51.

https://doi.org/10.1136/heartjnl-2016-310320

14. Alfonso Fernando, Bastante Teresa. Spontaneous Coronary Artery Dissection. Circ Cardiovasc Interv 2014 Oct 1;7(5):638-41. https://doi.org/10.1161/CIRCINTERVENTIONS.114.001984

15. Kwon T-G, Gulati R, Matsuzawa Y, Aoki T, Guddeti RR, Herrmann J, et al. Proliferation of Coronary Adventitial Vasa Vasorum in Patients With Spontaneous Coronary Artery Dissection. JACC Cardiovasc Imaging 2016 Jul 1;9(7):891-2. https://doi.org/10.1016/j.jcmg.2015.11.030

16. Meng P-N, Xu C, You W, Wu Z-M, Xie D-J, Zhang H, et al. Spontaneous Coronary Artery Dissection as a Cause of Acute Myocardial Infarction in Young Female Population: A Singlecenter Study. Chin Med J (Engl) 2017 Jul 5;130(13):1534-9. https://doi.org/10.4103/0366-6999.208245

17. Luong C, Starovoytov A, Heydari M, Sedlak T, Aymong E, Saw J. Clinical presentation of patients with spontaneous coronary artery dissection. Catheter Cardiovasc Interv 2017 Jun 1;89(7):1149-54. https://doi.org/10.1002/ccd.26977

18. Alfonso F, Paulo M, Lennie V, Dutary J, Bernardo E, JiménezQuevedo P, et al. Spontaneous Coronary Artery Dissection: Long-Term Follow-Up of a Large Series of Patients Prospectively Managed With a "Conservative" Therapeutic Strategy. JACC Cardiovase Interv 2012 Oct 1;5(10):1062-70.

https://doi.org/10.1016/j.jcin.2012.06.014

19. Sharma S, Raut N, Potdar A. Spontaneous coronary artery dissection: Case series and review of literature. Indian Heart J. 2016 Jul 1;68(4):480-5.

https://doi.org/10.1016/j.ihj.2015.11.039

20. Saw J, Mancini GBJ, Humphries K, Fung A, Boone R, Starovoytov A, et al. Angiographic appearance of spontaneous coronary artery dissection with intramural hematoma proven on intracoronary imaging. Catheter Cardiovasc Interv 2016;87(2):E54-61.

https://doi.org/10.1002/ccd.26022

21. Buccheri D, Zambelli G, Alfonso F, Cortese B. Pulse on Sponta- neous Coronary Artery Dissections: Experience-Based Survey. JACC Cardiovasc Interv [Internet]. 2017 Jul 24 [cited 2019 Dec 17];10(14):1469-71. Available from: http://www.sciencedirect. com/science/article/pii/S1936879817310786

https://doi.org/10.1016/j.jcin.2017.05.039

22. Rogowski S, Maeder MT, Weilenmann D, Haager PK, Ammann P, Rohner F, et al. Spontaneous Coronary Artery Dissection. Catheter Cardiovasc Interv [Internet]. 2017 [cited 2019 Dec 22];89(1):59-68. Available from: http://onlinelibrary.wiley. com/doi/abs/10.1002/ccd.26383

https://doi.org/10.1002/ccd.26383

23. Quadri G, Cerrato E, Escaned J, Rolfo C, Tomassini F, Ferrari F, et al. Importance of Close Surveillance of Patients With Conservatively Managed Spontaneous Coronary Artery Dissection. JACC Cardiovasc Interv [Internet]. 2018 Jun 11 [cited 2019 Dec 17];11(11):e87-9. Available from: http:/www.sciencedirect. com/science/article/pii/S1936879818307441

https://doi.org/10.1016/j.jcin.2018.03.020

24. Adlam D, Alfonso F, Maas A, Vrints C, Writing Committee. European Society of Cardiology, acute cardiovascular care association, SCAD study group: a position paper on spontaneous coronary artery dissection. Eur Heart J [Internet]. 2018 Feb 22 [cited 2019 Dec 22];39(36):3353-68. Available from: https://doi.org/10.1093/eurheartj/ehy080 https://doi.org/10.1093/eurheartj/ehy080

25. Rigatelli G, Barison S, Zuin M. Complex repair of coronary bifurcation spontaneous dissection. Asian Cardiovasc Thorac Ann [Internet]. 2019 Jul 1 [cited 2019 Dec 17];27(6):509-11. Available from: https://doi.org/10.1177/0218492318823363 https://doi.org/10.1177/0218492318823363

26. Pavlopoulos D, Hamilos M, Kalogerakos P, Kampitakis E, Tsipas P, Kokotsakis J, et al. Spontaneous Coronary Artery Dissection in a Woman: Surgical Treatment and Angiographic Follow-Up. Ann Thorac Surg [Internet]. 2018 Sep 1 [cited 2019 Dec 17];106(3):e133-5. Available from: http://www.sciencedirect.com/science/article/pii/S0003497518305022 https://doi.org/10.1016/j.athoracsur.2018.03.026

27. Motreff P, Ronchard T, Sanguineti F, Achkouty G, Benamer H, Range G, et al. Coronary Artery Fenestration. JACC Cardiovasc Interv 2018 Sep 24;11(18):1905.

https://doi.org/10.1016/j.jcin.2018.06.032

28. Tweet Marysia S, Hayes Sharonne N, Pitta Sridevi R, Simari Robert D, Lerman Amir, Lennon Ryan J, et al. Clinical Features, Management, and Prognosis of Spontaneous Coronary Artery Dissection. Circulation. 2012 Jul 31;126(5):579-88.

https://doi.org/10.1161/CIRCULATIONAHA.112.105718

29. Tweet Marysia S, Eleid Mackram F, Best Patricia JM, Lennon Ryan J, Lerman Amir, Rihal Charanjit S, et al. Spontaneous Coronary Artery Dissection. Circ Cardiovasc Interv. 2014 Dec 1;7(6):777-86.

https://doi.org/10.1161/CIRCINTERVENTIONS.114.001659 


\section{SPONTANINĖS KORONARINIŲ ARTERIJŲ DISEKACIJOS PATOGENEZĖS, DIAGNOSTIKOS IR VALDYMO APŽVALGA \\ A. Rèkus, G. Jaruševičius}

Raktažodžiai: vainikinių arterijų disekacija, spontaninis, ūmus koronarinis sindromas, intramuralinè hematoma.

Santrauka

Spontaninè vainikinių arterijų disekacija (SVAD) yra reta ūmaus išeminio sindromo priežastis. Pirmą kartą SVAD buvo aprašyta prieš 80 metų. Patogeneziniai mechanizmai greičiausiai yra susiję su įplèšimu ar kraujavimu vasa-vasorum, dẻl kurio atsiranda intramuralinis kraujavimas. SVAD dažniausiai nustatoma jaunoms moterims, neturinčioms koronarinès širdies ligos rizikos veiksnių ir kurioms yra ūminis koronarinis sindromas. Puse SVAD atvejų nustatomas miokardo infarktas su ST pakilimu, o likusiai daliai - be ST pakilimo. Auksinis standartinis diagnostinis metodas yra intervencinè vainikinių arterijų angiografija. Po ūminio išeminio sindromo daugumos pacientų klinikinè eiga yra stabili ir gerybiné, stebimas spontaninis kraujagyslių sienelių gijimas. Taigi, kaip pradini gydymo metodą rekomenduojama taikyti konservatyvų medicininį gydymą (budrus stebejjimas). Daugeliu atvejų intervencinis ir chirurginis gydymas atrodo suboptimalus. Esant tokiai nepaprastai sudètingai situacijai siūlomi keli nauji ir patrauklūs koronariniai intervenciniai gydymo metodai. Šioje apžvalgoje apibendrinami rizikos veiksniai, patogenezès teorijos, diagnostikos ir valdymo metodai bei SVAD prognozè.

Adresas susirašinėti: algirekus@gmail.com

Gauta 2020-02-18 